\title{
Singular perturbations in noisy dynamical systems $\dagger$
}

\author{
B. J. MATKOWSKY \\ Department of Engineering Sciences \& Applied Mathematics, \\ Northwestern University, Evanston, IL 60208, USA \\ email: b-matkowsky@northwestern.edu
}

(Received 21 November 2017; revised 15 January 2018; accepted 15 January 2018; first published online 31 January 2018)

\begin{abstract}
Consider a deterministic dynamical system in a domain containing a stable equilibrium, e.g., a particle in a potential well. The particle, independent of initial conditions, eventually reaches the bottom of the well. If however, the particle is subjected to white noise, due, e.g., to collisions with a population of smaller, lighter particles comprising the medium through which the particle travels, a dramatic difference in the behaviour of the Brownian particle occurs. The particle will exit the well. The natural questions then are how long will it take for it to exit and from where on the boundary of the domain of attraction of the deterministic equilibrium (the rim of the well) will it exit. We compute the mean first passage time to the boundary and the mean probabilities of the exit positions. When the noise is small each quantity satisfies a singularly perturbed deterministic boundary value problem. We treat the problem by the method of matched asymptotic expansions (MAE) and generalizations thereof. MAE has been used successfully to solve problems in many applications. However, there exist problems for which MAE does not suffice. Among these are problems exhibiting boundary layer resonance, i.e., the problem of 'spurious solutions', which led some to conclude that this was 'the failure of MAE'. We present a physical argument and four mathematical arguments to modify or augment MAE to make it successful. Finally, we discuss applications of the theory.
\end{abstract}

Key words: Singular perturbations, asymptotic expansions, JWKB methods.

\section{Introduction}

I am honoured and humbled by the award of the John von Neumann Lecture Prize by SIAM. This award is particularly meaningful to me, as four of the previous awardees were my teachers and my inspirations, including Kurt Friedrichs, Peter Lax, Jurgen Moser, and most importantly, my advisor Joe Keller. To have my name linked to theirs is a great honour indeed. Since two of these luminaries chose the subject of asymptotic expansions and applications thereof, for award talks, I have chosen to follow in their footsteps and speak on the same topic.

Asymptotics is the study of the local behaviour of a function. The function may be known a priori or we may only have hints as to what it is, e.g., that it satisfies a

$\dagger$ This paper corresponds to the von Neumann Lecture Prize awarded in 2017. 
differential equation (DE) and associated boundary and/or initial conditions. In this case, we employ a perturbation method to solve the problem. One such problem concerns the effect of a small perturbation, e.g., noise, on a deterministic dynamical system. If the perturbation leads to only a small effect on the system, we refer to this as a regular perturbation, whose result generally will be little noted nor long remembered. However, it is possible for the perturbation to have a dramatic effect, which is of far greater interest and is referred to as a singular perturbation (SP). This can occur, e.g., if the perturbation is random. The DE is then a stochastic DE, since certain terms are only known statistically, i.e., as stochastic processes, so that the solution is then only known statistically, as in a microscopic description of the phenomenon under consideration. However, certain macroscopic quantities, e.g., moments of the solution, may be shown to satisfy related deterministic DEs. To determine the local behaviour of the solution of the equation containing a small parameter, say $\epsilon$, i.e., the asymptotic solution locally in the neighbourhood of $\epsilon=0$, we employ SP techniques. Below, I will consider such problems.

Specifically, I will consider the exit problem. Thus, consider a deterministic dynamical system containing a stable equilibrium point, e.g., a particle in a potential well. The particle, no matter where it starts out, will eventually end up at the bottom of the well (the stable equilibrium point). If however, the particle is subjected to white noise (the 'derivative' of Brownian motion, with the quotes inserted since Brownian motion is nowhere differentiable and must be interpreted in terms of a stochastic process with an appropriate stochastic calculus, e.g., Ito or Stratonovich), due to collisions with a large population of smaller and lighter particles comprising the medium through which the Brownian particle travels, a dramatic difference in the behaviour of the particle can occur. Due to the collisions, the particle can exit the well, just as a gambler can have a run of good luck. It might take a long time, but it will, in fact, exit. To be sure, this occurrence is a rare event (not in the sense that its probability is low [it is 1], but rather that its frequency is low, which is why the exit time far exceeds the deterministic time scale). The natural questions then are (i) how long will it take for it to occur and (ii) from where on the boundary of the domain of attraction of the stable equilibrium point (the rim of the well) will it exit. These quantities are random variables. Thus, we will compute the mean first passage time (MFPT) to the boundary and the probability distribution of boundary points being exit points. Each quantity satisfies a deterministic boundary value problem (BVP) governed by a DE. When the noise is small, the resulting BVP is an SP problem.

We treat this problem by SP methods, in particular, the method of matched asymptotic expansions (MAE) and generalizations thereof. In 1904, L. Prandtl [44], in a talk presented at the International Congress of Mathematicians in Heidelberg, introduced a paper which revolutionized the study of fluid mechanics. In studying the low viscosity fluid flow over a solid object, he proposed that the viscous term in the Navier Stokes equations could be ignored in the fluid away from the solid, thus leading to the Euler equations, but must be accounted for in a thin layer near the boundary of the solid, where the solution varies rapidly. His theory came to be known as Boundary Layer theory. After hearing the talk, C. Felix Klein arranged a position for Prandtl in Gottingen, then the mecca of mathematics and science. Unfortunately, Prandtl, undoubtedly a great fluid mechanician, later showed himself to be a flawed human being as an apologist for the nazi regime. Boundary Layer theory was later systematized and generalized by K. O. Friedrichs and 
his student W. Wasow $[16,48]$ to what is now called MAE, which leads to an asymptotic series approximation to the solution of the SP problem under consideration. In the outer region (away from the boundary) the solution, $u$ is expanded in a power series in the small parameter, say $\epsilon$, with the coefficients functions of the independent variable, say $x$, which may be thought of as a spatial variable. Here, $\epsilon$ is a measure of the non-dimensional viscosity. In the boundary layer, the stretching transformation $\xi=\frac{x-x_{0}}{\epsilon^{\alpha}}$ is introduced, where $x_{0}$ denotes the location of the layer and $\alpha$ determines the width of the layer. The solution $u$ is then expanded in $\epsilon$ with coefficients functions of $\xi$, so that the solution is rapidly varying. Finally, the two expansions are appropriately matched so that they connect smoothly. It should be noted that in some problems, the thin layer is not attached to the boundary, but occurs in the interior of the domain, in which case it is referred to as an internal layer. We note that others have contributed to the development of MAE, notable among them N. Levinson [31] and his students, as well as researchers from the Soviet Union.

MAE has been used successfully to solve many problems in a wide variety of areas of application. However, there exist a number of problems for which MAE does not suffice. Among these is the phenomenon exhibiting so-called boundary layer resonance, i.e., the problem of 'spurious solutions'. In these problems, after all the conditions employed in MAE are applied, the asymptotic solution of the problem is not uniquely determined, as a constant is left undetermined. Thus, MAE yields a one parameter family of possible asymptotic solutions, though only one will turn out to be the solution. This led some researchers to conclude that they had encountered 'the failure of MAE'. Below, we present a physical argument and four different mathematical arguments to modify or augment MAE so that it is successful for such problems. Finally, we discuss applications of the above theory.

Since the lecture is intended for a large audience with many diverse specialties and interests, I have decided not to focus on technical details, but rather to focus on the basic ideas and present the material so that it is easy to follow. With this in mind, for ease of exposition, I will restrict attention to problems in one dimension, though results in higher dimensions [35-39,45], among others, have been obtained, as have results for non-linear problems [21], results for different types of noise, e.g., coloured noise [22,23] shot noise [3-5], Markovian and non-Markovian noise [10-12,24,25,40], as well as escape from a limit cycle [6]. In addition, I will restrict consideration to only the leading term in the expansion. First, some background material.

\section{Brownian motion}

In 1827, Robert Brown [8], a botanist, observed that when pollen grains were suspended in water, they moved in an agitated and irregular state of motion. He then showed that the same occurs for many types of fine particles, e.g., glass, metals. Interestingly, in 1785, a similar, though less systematic, observation was made by Jan Ingenhausz, a Dutch physiologist, of carbon dust particles suspended in alcohol. However, the phenomenon is known to this day as Brownian motion, perhaps because the physics community learned of it from Brown's work. Ingenhausz was the discoverer of photosynthesis, was the personal physician of Empress Maria Theresa of Austria, was a scientific correspondent 
of Benjamin Franklin and Henry Cavendish (for whom the Cavendish lab at Cambridge was named) and was a Fellow of the Royal Society. Thus, he was no slouch. This may be an example of Stigler's law of eponymy, which states that 'no discovery is named after its original discoverer'. We note that the path of the Brownian particle is not differentiable.

An explanation for Brownian motion was first provided by Einstein [14] in 1905. Interestingly, in this paper, he was unaware of the Brownian phenomenon. He was interested in the molecular composition of matter. It was not until 1906 that he discussed Brownian motion. However, the ideas for the explanation were already in the 1905 paper. Essentially, the same explanation was given in 1906, independently by Smoluchowski [46], both arguing that the motion was due to the frequent collisions of the Brownian particle with the smaller and lighter particles which are always on the move in the liquid in which they are suspended (there are $O\left(10^{21}\right)$ collisions/sec). The motion is so complicated that it can only be described probabilistically in terms of the very frequent, statistically independent collisions. This is because the individual collisions cannot be observed nor can the path of the Brownian particle, in any detail. We certainly cannot describe the motion of all the individual particles of the medium nor their interactions. This was the beginning of stochastic modelling of natural phenomena.

Einstein argued that there are two forces acting on the Brownian particle: (1) the collisions and (2) the viscous drag force on the Brownian particle in the fluid, so that the process is essentially a diffusive process (solution of $u_{t}=D u_{x x}$ ) and, using Stokes law for the viscous drag, computed the diffusion coefficient as

$$
D=\frac{k T}{6 \pi \eta a}
$$

where $k$ is Boltzmann's constant and $T$ the mean temperature. The viscous drag is $-6 \pi \eta a$, where $\eta$ is the drag coefficient and $a$ is the particle's diameter. Einstein formulated the diffusion equation whose diffusion coefficient was related to observable physical quantities. Thus, he was able to compute the size of the atoms and how many there were in a mole, i.e., Avogadro's number. His explanation gave definitive confirmation of the existence of atoms and molecules, which was then a topic of debate [Dalton and Boltzmann (pro) and Mach and Ostwald (anti)]. Einstein's explanation was experimentally verified by Perrin in 1908 [41] for which he received the Nobel prize.

Langevin in 1908 [29], employing the same two forces wrote the particle's equation of motion as

$$
m \ddot{x}=-6 \pi \eta a \dot{x}+R,
$$

where $R$ is a fluctuating (random) force modelling the collisions. He noticed the similarity of this process to a diffusion process and also concluded that

$$
D=\frac{k T}{6 \pi \eta a} .
$$

This was the first example of a stochastic DE.

Note that there is an intimate connection between probability (SDEs) and partial differential equations. As we saw, Brownian motion is associated with the diffusion equation, i.e., the probability density function is the probability that the Brownian particle, 
initially at the point $x_{0}$ will be at the point $x$ at time $t$, i.e., $p\left(x, t \mid x_{0}\right)$ satisfies the partial differential equation $p_{t}=D p_{x x}$. Thus, the particle will diffuse. For example, a drop of cream diffuses in a cup of coffee, as higher cream concentrations diffuse towards lower concentrations. The collision process is related to, and thus modelled by, diffusion. Since the process goes from $x_{0}$ at $t=s$ to $x$ at $t, x$ and $t$ are called the forward variables (where it is going), while $x_{0}$ and $s$ are called the backward variables (where it is coming from). The equations $p_{t}=L^{*} p \equiv p_{x x}$ and $p_{s}=L p$ satisfy the forward Kolmogorov [26] (a.k.a. Fokker [15]-Planck [42]) and Backward Kolmogorov equations for a free Brownian particle, respectively. Here, $L^{*}$ denotes the adjoint of the operator $L$. These equations will be employed below.

Henceforth, we will restrict attention to one dimension. The extension to higher dimensions will be clear. For purposes of illustration, we now employ a simpler, though not quite realistic, model of the collision process, to derive the diffusion equation. Specifically, we employ a random walk model on the line (1D) where the particle is located at point $x$ and can (a) jump to the right with probability $r(x)$, (b) jump to the left with probability $l(x)$ or $(\mathrm{c})$ not jump at all with probability $(1-r(x)-l(x))$. Let us assume that both the jump size $\epsilon$ and jump time $\Delta t$ are small. Thus,

$$
\begin{aligned}
& \operatorname{Prob}[\Delta x=\epsilon \mid x(t)=x]=r(x) \Delta t, \\
& \operatorname{Prob}[\Delta x=-\epsilon \mid x(t)=x]=l(x) \Delta t, \\
& \operatorname{Prob}[\Delta x=0 \mid x(t)=x]=(1-r(x)-l(x)) \Delta t,
\end{aligned}
$$

and let $p(x, y, t)$ denote the probability of reaching $x(t)=y$ given $x(0)=x$, with $y$ the forward variable and $x$ the backward variable. Then,

$$
\begin{aligned}
p(x, y, t)= & p(x, y-\epsilon, t-\Delta t) r(y-\epsilon) \Delta t+p(x, y+\epsilon, t-\Delta t) l(y+\epsilon) \Delta t \\
& +p(x, y, t-\Delta t)[1-r(y)-l(y)] \Delta t .
\end{aligned}
$$

Now, consider

$$
\frac{p(x, y, t)-p(x, y, t-\Delta t)}{\Delta t} .
$$

Expand in $\epsilon$ and take the limit as $\Delta t$ goes to 0 to obtain

$$
\frac{\partial p}{\partial t}=L^{*} p=\epsilon \frac{\partial}{\partial y}[(l(x)-r(x)) p]+\frac{\epsilon^{2}}{2} \frac{\partial^{2}}{\partial y^{2}}[(r(x)+l(x)) p]+O\left(\epsilon^{3}\right) .
$$

Define $\tau=\epsilon t$, so that the equation describes behaviour for large times, since for short or even $O(1)$ times there is hardly any movement. After ignoring the $\mathrm{O}\left(\epsilon^{3}\right)$ term and cancelling the factor $\epsilon$, which is common to all terms, we arrive at

$$
\frac{\partial p}{\partial \tau}=-\frac{\partial[(r-l) p]}{\partial y}+\frac{\epsilon}{2} \frac{\partial^{2}((r+l) p)}{\partial y^{2}},
$$

a diffusion equation.

The first term on the right side of this equation is called the drift, while the second term describes diffusion. For example, if $r>l$, there is a bias of jumping towards the right, while if $l>r$, there is a bias of jumping towards the left. 
We see that if $r=l$, the drift term vanishes and we have

$$
\frac{\partial p}{\partial \tau}=\epsilon \frac{\partial^{2}}{\partial y^{2}}(r p),
$$

which describes a pure diffusion on the long time scale. If $r \neq l$, there is a drift so that it describes diffusion in a force field. Note that $\epsilon$ multiplies the diffusion term so that it may be thought of as studying the effect of perturbing the drift, which might model a noisy perturbation of a deterministic dynamical system. This is an SP problem. The random walk description may be thought of as a description at the microscopic level, while the diffusion equation may be thought of as a description at the macroscopic level. This can be extended to account for long jumps and for waiting times before jumping.

In his book 'Stochastic Processes in Physics and Chemistry', N. von Kampen [47] asks 'why do stochastic processes enter into physics?' He answers 'many phenomena which evolve in time in an extremely complicated way, well beyond any possibility of calculation or even observation, do have some average features that can be observed and obey simple laws. For example, the precise instantaneous value of the force exerted by the molecules of a gas medium on a piston over a time interval becomes a smooth function obeying Boyle's law. The use of probability considerations is justified by our ignorance of the precise microscopic state. However, in spite of our ignorance of the microscopic details, macroscopic variables are observable and can be calculated'.

It was Norbert Wiener [50], who provided a formalized mathematical theory of Brownian motion in terms of what is now called a Wiener process $w$. Its 'derivative' is referred to as white noise, which is a model for the random collision process. The reason for the quotes around the word derivative is that the path of the Brownian particle is not, of course, differentiable. White noise is only a caricature of a real noise process since its power spectral density, i.e., the area under the curve of power (a measure of the amplitude, e.g., the $L_{2}$ norm of $x(t)$ which satisfies $\|x(t)\|_{L_{2}}=\left\|x_{f}(\omega)\right\|_{L_{2}}$, with $x_{f}$ the Fourier transform of $x$ ) vs. the frequency $\omega$, is infinite. A more realistic model is the so called coloured noise, whose power spectral density is constant for finite $\omega$, but tails off for large $\omega$, and therefore has a finite power spectral density. Nevertheless, white noise is quite useful for the description of Brownian motion type phenomena.

\section{Stochastic differential equations (SDEs)}

An SDE is a DE containing term(s) which are only known probabilistically. Thus, the solution is only known probabilistically. Examples include phenomena which involve many collisions at the microscopic level, e.g., Brownian motion. Here, we shall not study the details of manipulating SDEs, since dealing with the details of the microscopic behaviour are generally sufficiently complicated to calculate and often even to observe. Rather, we will consider certain partial differential equations which are related to the SDEs and compute certain macroscopic quantities which are both calculable and observable. We will employ SP techniques, in particular the method of MAE, to compute these quantities. It should be mentioned that employing SP techniques in these problems began in the 1970s by Matkowsky and colleagues, and was continued for years thereafter. 
We consider a deterministic dynamical system of the form $\dot{x}=b(x)$, where $x(t)$ may be a vector. We perturb the system by small noise of the form considered above, i.e., white noise. Thus, e.g., we consider the SDE

$$
d x=b(x) d t+\sqrt{2 \epsilon} d w,
$$

where $\sqrt{2 \epsilon}$ is the amplitude, here a small constant, and $d w$ describes white noise. As noted above, the Wiener process $w$ and the white noise process $d w$ are not differentiable, so that the white noise description must be defined appropriately. It might seem better to integrate the SDE to obtain a stochastic integral equation. However, it would then be necessary to define the resulting stochastic integral of the random term. Without going into technical detail, there are two widely used stochastic integrals. The Ito integral and the Stratonovich integral. The result is the same for both provided the noise term is state independent, i.e., independent of $x$, e.g., the amplitude is constant as above. However, they may differ if the noise is state dependent. Here we consider state independent white noise of amplitude $\sqrt{2 \epsilon}$ (with $\epsilon$ denoting the small parameter $k T$ ), as in Brownian motion. We will not use the stochastic integral equation approach here.

Thus, we consider the SDE

$$
d x=b(x) d t+\sqrt{2 \epsilon} d w
$$

where the first term describes the deterministic drift and the second the random term, describing diffusion due to the noise.

In contrast to the deterministic situation, where we can predict the location and velocity of a particle at time $t$, knowing the forces acting on the particle and its initial conditions, due to noise we can no longer know its exact position or velocity. We can only determine the probabilities of being at a certain position $x$, and having a certain velocity at time $t$. These are random quantities.

For the SDE above, the corresponding stationary (time-independent) Kolmogorov forward operator is

$$
L^{*} p=-(b p)_{y}+\epsilon p_{y y},
$$

where $y$ is the forward variable, while the stationary Kolmogorov backward operator is

$$
L p=b p_{x}+\epsilon p_{x x},
$$

where $x$ is the backward variable. The Kolmogorov forward equation describes the time evolution of the probability distribution function. The Kolmogorov backward equation can be employed to compute the MFPT and also the distribution of the exit points on the boundary, in the exit problem discussed in the next section.

\section{The exit problem}

If the effect of small noise is to change the behaviour of the deterministic dynamics by only a small amount, it is of little interest. However, the effect can be quite dramatic. For example, consider a deterministic system with a stable attractor in a domain $D$, say at the origin. Assume that the dynamic forces are derivable from a potential $V(x)$. 
We show below that due to even very small noise, the particle is guaranteed to leave (exit) the domain of attraction $D$, in which it initially exists. The occurrence of exit is analogous to a gamblers run of good luck. Both events are examples of rare events, occurring in the tails of the probability distribution. They are also dealt with in the theory of large deviations. It might take a very long time to leave, but it will leave. In fact, the MFPT can be shown to be $O\left(e^{1 / \epsilon}\right)$. Incidentally, independent of any computations, it would seem to be intuitively clear that the point(s) on the boundary at which the particle exits should have the shortest exit path. Thus, if there is one such point, the probability of exiting there is 1 , and the probability of exiting elsewhere on the boundary is zero. If there are $N$ such points, the probability of exiting from each is $\frac{1}{N}$, and zero elsewhere, since the probability of exiting from somewhere is 1 .

The so called exit problem consists in determining (1) how long will it take to exit the domain $D$ and (2) from where on the boundary of $D$ will the particle exit? Specifically, (1) what is the MFPT from $D$ and (2) what is the probability of each boundary point being an exit point? Note that if we ran the experiment multiple times, or had multiple experimenters run the experiment, we would not get the same outcome every time. Thus, it is a random variable, and we can only determine statistics, e.g., its mean.

We first consider the problem of a free Brownian particle in one dimension. That is, there are no additional forces acting on the particle $(b(x) \equiv 0)$. Let the domain $D$ be the interval $(-a, b)$, with the constants $a, b>0$ and $x=0$ is a stable equilibrium point.

The MFPT $\tau$ is a solution of the BVP

$$
\begin{aligned}
L \tau & =\epsilon \tau^{\prime \prime}=-1 \quad \text { in } D, \\
\tau & =0 \quad \text { on } \partial D
\end{aligned}
$$

where $L$ is the stationary backward Kolmogorov operator $\epsilon \tau^{\prime \prime}$ (see Dynkin [13]). It follows from Ito's formula [19], as we now show. Let $x_{\epsilon}$ be the solution of the more general SDE for a particle starting at $x$

$$
d x_{\epsilon}=b\left(x_{\epsilon}\right) d t+\sqrt{2 \epsilon} d w,
$$

where $b$ is the drift, $\sqrt{2 \epsilon}$ the diffusion coefficient and $d w$ denotes white noise. Then, the stationary backward Kolmogorov operator $L$ is

$$
L=\epsilon \frac{\partial^{2}}{\partial x^{2}}+b \frac{\partial}{\partial x}
$$

and the operator $M$ acting on $f$ is $M f=\frac{\partial f}{\partial x}$. The Ito formula in integral form, for any sufficiently smooth function $f$, is then given by

$$
f\left(x_{\epsilon}\right)=f(x)+\int_{0}^{t} L f\left(x_{\epsilon}\right) d s+\int_{0}^{t} M f d w,
$$

the latter term being a stochastic integral. Let $u$ be the solution of the BVP $L u=-1$ in the domain $D$ and boundary condition $u=0$ on $\partial D$, where $D$ is the interval $(-a, b)$ with $a, b>0$. Let $f=u$ and let $t=T$ in Ito's formula, where $T$ is the first passage time to $\partial D$, a random variable. Then, since $L u=-1$ in $D$ and $u=0$ on $\partial D$, we have that $u(x)-T+\int_{0}^{T} M u d w=0$. Finally, taking the expectation of the equation, and using the 
fact that the expectation of a stochastic integral vanishes, we arrive at $u(x)=E T=\tau$, where $E$ denotes expectation and $\tau$ is the MFPT.

The boundary condition is clear since the time to reach the boundary is zero if you start at the boundary. The solution of the BVP with $b(x)=0$ is $\tau=\frac{(a+x)(b-x)}{2 \epsilon}$. Thus, for any fixed $x$ in $D$, the particle will exit in finite time. Note that the MFPT is algebraically large in $\epsilon$.

Similarly, we show below that $\tau$ is finite, so exit is guaranteed, for Brownian particles when external forces are present $(b(x) \neq 0)$. For example, a particle in a potential well, e.g., $b(x)=-x$, with the origin as a deterministically stable equilibrium point. Here, $\tau$ is a solution of the BVP

$$
\begin{aligned}
L \tau & =\epsilon \tau^{\prime \prime}-x \tau^{\prime}=-1 \quad \text { in } D, \\
\tau & =0 \quad \text { on } \partial D,
\end{aligned}
$$

corresponding to a particle in the potential well $V(x)=x^{2} / 2$, so that the force is the drift term $b(x)=-x$. Let the domain $D$ be the interval $(-a, b)$, with $a, b>0$. The well exerts a force which constrains the particle to remain in the well. Since the solution of the above BVP is finite for any $\epsilon>0, \tau$ is finite, so exit occurs in finite time. Alternatively, we can let $f=w=1$ in Ito's formula. Thus, $L w=0$ in $D, w=1$ on $\partial D$. Then, let $t=T$, take the expectation and use the fact that the expectation of a stochastic integral vanishes. We find that the probability of a particle starting at a point $x$ in $D$ reaching a point on the boundary $\partial D$ is 1 , which states that exit occurs with probability 1 . In fact, it is known that the solution $\tau$ is $O\left(e^{\frac{1}{\epsilon}}\right)$ [35]. Thus, even for a small $(O(\epsilon))$ noise perturbation, the particle will certainly exit, though it will take a long time to do so. Here, the MFPT is exponentially large in $\epsilon$, which is reasonable since it takes much longer to exit when the particle has to overcome the constraining force of the potential. Another way to characterize $\tau$ is to note that $\tau=1 / \lambda$, where $\lambda$ is the second eigenvalue of the stationary Fokker-Planck (forward Kolmogorov) operator [36].

We next consider the probability distribution of exit points on the boundary $v(x)$ for a Brownian particle in a well. It is a solution of the stationary backward Kolmogorov BVP with associated boundary conditions

$$
\begin{aligned}
L v=0 & \text { in } D, \\
v=\phi & \text { on } \partial D,
\end{aligned}
$$

where $\phi$ describes the boundary data. Now, we let $f=v$ and let $t=T$ in Ito's formula, as above. Following the derivation of the BVP for $\tau$, we have that

$$
v\left(x_{\epsilon(T)}\right)=v(x)+\int_{0}^{T} M v d w .
$$

Again, taking the expectation of the equation, we arrive at

$$
E\left(x_{\epsilon(T)}\right)=v(x)
$$


where $E$ denotes expectation. In general, therefore,

$$
v(x)=\int_{\partial D} \phi(y) \rho(x, y) d S_{y},
$$

where $\rho(x, y)$ is the probability density of a particle starting at a point $x$ in $D$, is obtained as the Green's function of the Dirichlet problem above. In our case, the integral reduces to the sum of the contributions of the two boundary values, so that

$$
v=P_{-a} \alpha+P_{b} \beta,
$$

where $P_{-a}$ and $P_{b}$ are the probabilities of exiting at $-a$ and $b$, respectively. Intuitively, we expect that $P_{-a}=1$ and $P_{b}=0$, if $a<b$, i.e., if the point $x=-a$ is closer to the deterministically stable equilibrium point at the origin, so that $x=-a$ will be the exit point, while $P_{b}=1$ and $P_{-a}=0$ if $a>b$. Finally, $P_{b}=P_{-a}=\frac{1}{2}$ if $b=a$, so that both boundary points are equally likely to be the exit point. Intuitively, the exit point should correspond to the shortest distance from the equilibrium to the boundary. However, intuitive reasoning is not a mathematical demonstration. Below, we will see that this result is in fact true, by explicit calculation of the solution of the appropriate BVP. Thus, we will show that our intuitive expectation will be confirmed. We note that in addition to escape from an equilibrium, we have considered escape from a stable limit cycle [6], escape problems in higher dimensions [35-39,45] to name but a few and escape due to different types of noise, e.g., coloured noise [22,23], shot noise [3-5], Markovian and non-Markovian noise [10-12,24,25,40].

\section{Probability distribution of exit points}

In this section, we solve for the stationary probability distribution of the exit points on the boundary for a particle in a potential well. In this case, the boundary points are $x=-a$ and $x=b$. Thus, we consider the BVP

$$
\begin{aligned}
L u & =\epsilon u^{\prime \prime}-x u^{\prime}=0 \quad \text { in } D, \\
u(-a) & =\alpha, \quad u(b)=\beta .
\end{aligned}
$$

We first employ the method of MAE. That is, we first consider the outer expansion, which is to be valid in the interior of the domain $-a<x<b$, but not near at least one, and possibly both, of the boundaries. We assume that the outer solution has the asymptotic representation $u \sim \sum_{j=0}^{N} u_{j}(x) \epsilon^{j}$. We then insert this ansatz into the equation and boundary conditions and equate to zero the coefficient of each power of $\epsilon$ separately, thus obtaining a sequence of BVPs for the recursive determination of the coefficients $u_{j}$ in the expansion. In this way, we find that each coefficient is constant, $c_{0}, c_{1}, c_{2}, \ldots$, with the $c_{j}$ as yet undetermined. Note that since the reduced equation, i.e., the equation with $\epsilon=0$, satisfied by the leading term of the expansion, is first order, it cannot possibly satisfy both boundary conditions, in general. Thus, there must be a boundary layer at one or the other boundary point, or possibly even at both. We next construct possible boundary layer functions by stretching. Near $x=-a$, the stretched variable is $\xi=\frac{x+a}{\epsilon}>0$ 
and near $x=b$, it is $\eta=\frac{b-x}{\epsilon}>0$. We find that the left boundary layer function is $A+B e^{-a \xi}$ and the right boundary layer function is $C+D e^{-b \eta}$, each of which rapidly decays to a constant as we approach the interior of the domain. Applying the boundary conditions and matching the boundary layer solutions to the outer expansion, we obtain the leading term of the composite expansion, which is to be valid uniformly throughout the interval as

$$
u_{0}=c_{0}+\left(\alpha-c_{0}\right) e^{-a \xi}+\left(\beta-c_{0}\right) e^{-b \eta}
$$

But, what is $c_{0}$ ? Going to higher orders in $\epsilon$ does not help to determine $c_{0}$. Thus, though the exact solution is unique, we have not obtained a unique asymptotic representation of the solution. Rather, we have obtained a one parameter family of possible solutions, with no way to pick the correct member of the family, though we have used all the conditions of the MAE method. We note that if $c_{0}=\alpha$ the left boundary layer vanishes, if $c_{0}=\beta$ the right boundary layer vanishes and if $c_{0} \neq \alpha, \beta$ neither boundary layer vanishes. To summarize, we neither know what $c_{0}$ is, nor do we know whether there are one or two boundary layers, and if one, whether it is the left or right layer. The inability to determine a unique asymptotic representation of the solution was interpreted as a failure of the method of MAE. The class of problems to which this problem belongs exhibits the so called boundary layer resonance phenomenon, i.e., the problem of 'spurious solutions', originally described by Ackerberg and O’Malley [1]. In 1975, Matkowsky [34] formally gave a sequence of condition for boundary layer resonance to occur, which were then shown to be both necessary and sufficient. The phenomenon of MAE leading to a one parameter family of possible asymptotic solutions has also appeared in non-linear problems, e.g., [21].

To resolve the question of determining $c_{0}$, a number of proposals have been introduced. Here, we describe four such approaches. We note that all four approaches yield the same values of $c_{0}$. These methods are:

(i) In 1975, Matkowsky [34] employed a different way to construct the boundary layer function than in MAE, specifically the JWKB method [7,20,28,49]. In this way, he was able to find $c_{0}$ and in one case found that a single boundary layer function sufficed to describe two separate boundary layers. As above, the leading term of the outer expansion is $c_{0}$, which cannot possibly satisfy both boundary conditions (unless, as a special case, $\alpha=\beta$ ). Thus, it is necessary to construct a boundary layer function at one or the other end point, or possibly two separate boundary layer functions. Rather than construct two separate boundary layer functions as in the method of MAE, he employed the JWKB method to construct a single boundary layer function. Thus, he sought a solution in the form $u \sim A(x) e^{\frac{-\phi(x)}{\epsilon}}$. He inserted this ansatz into the DE and equating to zero the coefficient of each power of $\epsilon$ separately, to obtain the eikonal equation for $\phi$ as $\left(\phi^{\prime}\right)^{2}+x \phi^{\prime}=0$. There are two solutions: $\phi^{\prime}=0$ and $\phi^{\prime}=-x$. The former leads to the outer expansion, while the latter corresponds to $\phi=\frac{K^{2}-x^{2}}{2}$, with the constant $K$ chosen so that $\phi>0$ throughout the interior of the domain, $\phi=0$ at the boundary in order that the boundary conditions are satisfied and the boundary layer function decays rapidly away from the boundary. Thus, he took $K=\max (a, b)$. To leading order, the function $A(x)$ is a solution of the transport equation $x A^{\prime}+A=0$, whose solution is $A=\frac{a_{0}}{x}$ (with the - sign incorporated into $a_{0}$ ). 
Note that as $x \rightarrow-a, \phi \rightarrow a(x+a)$, while as $x \rightarrow b, \phi \rightarrow b(b-x)$, i.e., the standard MAE construction.

He then constructed the uniform expansion, valid throughout the interval $-a \leqslant x \leqslant b$ as

$$
u \sim c_{0}+\frac{a_{0}}{x} e^{-\frac{\left(K^{2}-x^{2}\right)}{2 \epsilon}}
$$

He considered the three cases separately.

In case $1(a<b)$ so that $K=b$, the uniform expansion is given by

$$
u \sim c_{0}+\frac{a_{0}}{x} e^{-\frac{\left(b^{2}-x^{2}\right)}{2 \epsilon}}
$$

Applying the boundary conditions, and ignoring transcendentally small terms, he obtained two equations for the constants $c_{0}$ and $a_{0}$

$$
\begin{gathered}
c_{0}=\alpha, \\
c_{0}+\frac{a_{0}}{b}=\beta .
\end{gathered}
$$

Here, the left boundary layer vanishes, and only one boundary layer remains at $x=b$. In case $2(a>b)$ so that $K=a$, the uniform expansion is

$$
u \sim c_{0}+\frac{a_{0}}{x} e^{-\frac{\left(a^{2}-x^{2}\right)}{2 \epsilon}}
$$

Applying the boundary conditions, and ignoring transcendentally small terms, he obtained

$$
\begin{gathered}
c_{0}-\frac{a_{0}}{a}=\alpha, \\
c_{0}=\beta .
\end{gathered}
$$

Here, the right boundary layer vanishes, and only one boundary layer remains at $x=-a$. Finally, in case $3(\mathrm{a}=\mathrm{b})$, the uniform expansion is the same as either of the above so that using the boundary conditions he obtained

$$
\begin{aligned}
& c_{0}-\frac{a_{0}}{a}=\alpha, \\
& c_{0}+\frac{a_{0}}{a}=\beta .
\end{aligned}
$$

Thus,

$$
c_{0}=\frac{\alpha+\beta}{2}
$$

so that both boundary layers remain. These results agree with those predicted intuitively above.

Note that the construction with a single boundary layer function suffices to describe two separate boundary layers when $a=b$. We also note that $A$ has a singularity that, in fact, has no effect on the solution since the boundary layer function has an effect only near the boundary or boundaries, and not in the interior of the interval. We can introduce a mollifier, a la Friedrichs. That is, in the immediate neighbourhood of the origin, we 
can replace the singularity by any smooth extension of the behaviour on either side of the singularity, thus getting rid of the singularity. In any case, it has no effect on the behaviour at the origin, since it is asymptotically negligible in the interior of the domain.

(ii) In 1975, Grasman and Matkowsky [17] introduced a variational problem whose Euler-Lagrange equation is the given DE, and used the family of functions determined by MAE, as the class of admissible functions for the variational problem. By equating the first variation to zero and then asymptotically expanding the resulting integrals, they found that $c_{0}=\alpha$ if $a<b, c_{0}=\beta$ if $a>b$ and $c_{0}=\frac{\alpha+\beta}{2}$ if $a=b$, which is exactly what was predicted intuitively, and agrees with the result obtained in approach (i).

(iii) In 1977, Matkowsky and Schuss [35] introduced an extension of the method of Grasman and Matkowsky to consider both problems having a variational formulation as well as those not having a variational formulation. In addition, they considered the problem not only in one dimension, but in higher dimensions as well. After employing MAE to determine the one parameter family of possible asymptotic expansions of the solution of the BVP for $u$ as above, they employed the following condition to determine $c_{0}$, rather than the variational condition. Specifically, they used the condition

$$
\left(p^{s}, L u\right)=0,
$$

where $L^{*} p^{s}=0$ is the stationary Fokker-Planck (forward Kolmogorov) equation, so that for our problem the stationary distribution $p^{s}=C e^{-\frac{x^{2}}{2 \epsilon}}$, where $C$ is a normalizing constant. They next integrated by parts, employed Green's second identity and asymptotically evaluated the integral. The condition above involves the inner product $(f, g)=\int_{-a}^{b} f g d x$ for the functions $f$ and $g$. It may be thought of as that employed in the Galerkin method, whereas the variational condition may be thought as that employed in the Ritz method. Retaining only the leading term in the expansion and employing this function $p^{s}$ and the one parameter family determined by MAE for $u$ in the condition above, they derived the very same values for $c_{0}$ as in the two methods above.

(iv) Finally, Chapman and Matkowsky [9] in unpublished work in 2013, considered the method of asymptotics beyond all orders (exponential asymptotics) to determine $c_{0}$. After the completion of this work, they learned of the work of MacGillivray [32] which proposed essentially the same idea for a related problem. We next describe our approach, after a short introduction.

Poincare in 1886 [43] defined an asymptotic series expansion with respect to an asymptotic sequence $\left\{\phi_{n}(\epsilon)\right\}$, e.g., $\left\{\epsilon^{n}\right\}$, with $\phi_{n+1}=o\left(\phi_{n}\right)$. Often the sequence, and corresponding expansion are sufficient to provide an accurate approximate solution of the problem under consideration. However, the question naturally arises: are there enough elements in the sequence for this strategy to succeed? That is, are they complete, i.e., does the sequence span the solution space for the problem, just as we ask in Fourier series? For many problems, the answer is affirmative. However, if we encounter a problem where the answer is negative, it will then be necessary to include additional term(s) to the sequence which 
are beyond all orders in $\epsilon$, e.g., terms which are exponentially small, $\left(O\left(e^{\frac{-k}{\epsilon}}\right)\right)$. Thus, the terms asymptotics beyond all orders and exponential asymptotics were born. Here, our problem is just such a problem. Of course, we note that we have already encountered the need to include exponentially small terms when introducing boundary layers.

Since we were not able to determine $c_{0}$ above by MAE, we see that the asymptotic sequence $\left\{\epsilon^{n}\right\}$ does not appear to be sufficient to span the solution space. We suspect that it is necessary to consider asymptotics beyond all orders, otherwise referred to as exponential asymptotics. Thus, in the outer region (interior of the domain), we include terms not yet included in the asymptotic expansion, i.e., which are $O\left(e^{-1 / \epsilon}\right)$. Then, together with the original asymptotic series, they must match the existing boundary layer series. Thus, we employ the JWKB method to construct an exponentially small solution in the interior of the domain. This is in contrast to introducing the JWKB construction in the boundary layer(s) in approach (i). We seek such a solution in the form $u=w e^{\frac{\phi(x)}{\epsilon}}$, with $w \sim w^{0}+\epsilon w^{1}+\cdots$. After inserting the ansatz into the equation and equating to zero, the coefficient of each power of $\epsilon$ separately, we find that $\phi$ satisfies the eikonal equation

$$
\left(\phi^{\prime}\right)^{2}-x \phi^{\prime}=0
$$

and the leading term $w^{0}$ satisfies the transport equation

$$
\left(2 \phi^{\prime}-x\right)\left(w^{0}\right)^{\prime}+\phi^{\prime \prime} w^{0}=0 .
$$

The eikonal equation has two solutions: $\phi^{\prime}=0$ and $\phi^{\prime}=x$. The former is constant and can be ignored. The solution of $\phi^{\prime}=x$ corresponds to $\phi=\frac{x^{2}}{2}+C$, and we can take $C=0$. Then, using $\phi^{\prime}=x$, we find that $w^{0}=\frac{K}{x}$, with $K$ constant. We note that $w_{0}$ has a singularity at $x=0$. Therefore, we construct an internal layer at $x=0$. Thus, the JWKB solution will be valid separately in each of the two regions, $x<0$ and $x>0$, but not at $x=0$. We write these as $u_{-}^{0}=\frac{K_{-}}{x} e^{\left(\frac{x^{2}}{2 \epsilon}\right)}$ and $u_{+}^{0}=\frac{K_{+}}{x} e^{\left(\frac{x^{2}}{2 \epsilon}\right)}$, respectively. These appear to be exponentially large, but we shall see, after calculating $K_{-}$and $K_{+}$, that they are actually asymptotically small. To construct the internal layer solution, we introduce the stretching transformation $\xi=\frac{x}{\sqrt{\epsilon}}$, and let $u(x) \rightarrow U(\xi)$, to obtain the leading order internal layer equation

$$
U_{\xi \xi}^{0}-\xi U_{\xi}^{0}=0, \quad-\infty<\xi<\infty
$$

whose solution is

$$
U^{0}=\gamma_{1} \int_{0}^{\xi} e^{\frac{s^{2}}{2}} d s+\gamma_{2},
$$

with $\gamma_{1,2}$ constant. Multiply and divide by $s$, then integrate by parts to obtain

$$
U^{0}=\gamma_{1} \frac{e^{\frac{\xi^{2}}{2}}}{\xi}+\gamma_{1} \int_{0}^{\xi} \frac{e^{\frac{s^{2}}{2}}}{s^{2}} d s+\gamma_{2}
$$

Note that as $\xi \rightarrow \infty$, the first term clearly dominates the others. 
This internal layer solution as $\xi \rightarrow \infty$ must match the right outer solution $u_{+}^{0}$ (including its exponentially small part) as $x \rightarrow 0$, so that

$$
\frac{\sqrt{\epsilon} \gamma_{1}}{x} e^{\frac{x^{2}}{2 \epsilon}}=\frac{K_{+}}{x} e^{\frac{x^{2}}{2 \epsilon}}
$$

Thus, $K_{+}=\sqrt{\epsilon} \gamma_{1}$. Similarly, matching the internal layer solution as $\xi \rightarrow-\infty$, to the exponentially small left outer solution $u_{-}^{0}$ as $x \rightarrow 0$, we have that $K_{-}=-\sqrt{\epsilon} \gamma_{1}$. Thus, $K_{-}=-K_{+}$.

Next, we match the outer solution (including its exponentially small part) to the left boundary layer solution. The outer solution is given by

$$
c_{0}+\frac{K_{-}}{x} e^{\frac{x^{2}}{2}}
$$

written to leading order in terms of $\xi$ near the left boundary as

$$
c_{0}-\frac{K_{-}}{a} e^{\frac{a^{2}}{2 \epsilon}} e^{-a \xi} .
$$

The left boundary layer solution is given by

$$
c_{0}+\left(\alpha-c_{0}\right) e^{-a \xi}
$$

After matching, we obtain

$$
K_{-}=a\left(c_{0}-\alpha\right) e^{-\frac{a^{2}}{2 \epsilon}} .
$$

Note that $K_{-}$is indeed exponentially small in $\epsilon$, which implies that $u_{-}^{0}$ is exponentially small.

Similarly, matching the right outer solution (including its exponentially small part) to the right boundary layer solution, and using $K_{-}=-K_{+}$, we obtain

$$
K_{-}=b\left(\beta-c_{0}\right) e^{-\frac{b^{2}}{2 \epsilon}} .
$$

Equating the two expressions for $K_{-}$, we have that to leading order

$$
c_{0}=\frac{a \alpha e^{\frac{-a^{2}}{2 \epsilon}}+b \beta e^{\frac{-b^{2}}{2 \epsilon}}}{a e^{\frac{-a^{2}}{2 \epsilon}}+b e^{\frac{-b^{2}}{2 \epsilon}}} .
$$

We now analyse this expression for each of the three cases: $a<b, a>b$ and $a=b$.

case 1: ignore $e^{\frac{-b^{2}}{2 \epsilon}}$ terms since they are asymptotically smaller, so that $c_{0}=\alpha$,

case 2: ignore $e^{\frac{-a^{2}}{2 \epsilon}}$ terms since they are asymptotically smaller, so that $c_{0}=\beta$, finally

case 3 : keep all terms, so that $c_{0}=\frac{\alpha+\beta}{2}$, exactly as intuitively predicted, and obtain the same results as in (i), (ii) and (iii).

Again, as in approach (i), there is an apparent singularity at the origin, which can be taken care of by the introduction of a mollifier, as above. Since the exponentially small term introduced into the interior of the domain is only needed near the boundaries, in 
order to match to the boundary layer expansion(s), it is asymptotically ignorable at the origin, as above.

There is one more item to take care of. The solution is expected to depend continuously on the data of the problem, in this case $a$ and $b$. However, it does not appear to do so, since for $a \neq b, c_{0}=\alpha$ or $\beta$, according as $a<b$ or $a>b$, whereas for $a=b, c_{0}=\frac{\alpha+\beta}{2}$. As it now stands a very small change in the boundary locations can cause an $O(1)$ change in the solution. This is due to the fact that we only considered $b-a$ to be an $O(1)$ quantity. Thus, we now consider $a$ and $b$ to differ by only a small amount. Specifically, we let $b=a+\epsilon d$ to derive a formula which does depend continuously on the data and bridges the gap between the various results for $c_{0}$. We employ $b=a+\epsilon d$ in the formula above involving the exponentials $e^{\frac{-a^{2}}{2 \epsilon}}$ and $e^{\frac{-b^{2}}{2 \epsilon}}$ to get

$$
c_{0}=\frac{\alpha+\beta e^{-a d}}{1+e^{-a d}}=\frac{\alpha+\beta e^{-a \frac{b-a}{\epsilon}}}{1+e^{-a \frac{b-a}{\epsilon}}} .
$$

Clearly, as $d \rightarrow \infty$, we get $c_{0}=\alpha$, as $d \rightarrow-\infty$, we get $c_{0}=\beta$ and finally, if $d=0$, we get $c_{0}=\frac{\alpha+\beta}{2}$. Thus, the formula depends continuously on the data, and serves as a bridge between the various cases. Note that this indicates that exit does not correspond to the isolated value $c_{0}=\frac{\alpha+\beta}{2}$, but rather to a narrow distribution about that value. The considerations above were for one dimension. Extensions to higher dimensions as well as to various types of noise and to applications were considered by Matkowsky and colleagues.

We note that there is another approach to the problem [30] which, motivated by the discussion in [21], involves projection onto the eigenspace associated with the exponentially small eigenvalue that appears in the problem.

\section{Kramers model of chemical reaction rates: Brownian particle in a field of force}

As an application of the above theory, consider the following. In 1940, Kramers [27] considered Brownian motion in a field of force, e.g., in a potential well, which, due to collisions with the particles in the medium in which it exists, escapes the well. He used this as a model for studying chemical reaction rates. Reaction occurs when the particle overcomes the potential barrier, whose height is $E$, and leaves the well.

Kramers studied the forward Kolmogorov equation to derive a formula for the reaction rate. In contrast to Kramers, we consider the backward Kolmogorov equation. The rate of escape $\kappa$ from the well is inversely proportional to the time to escape the well. Thus, $\kappa$ is given by

$$
\kappa=\frac{1}{2 \tau},
$$

where $\tau$ denotes the MFPT, i.e., the mean time to reach the top of the potential barrier and the factor $\frac{1}{2}$ is due to the fact that it is then equally likely to escape or return to the well. Therefore, by calculating the MFPT from the well, we have the escape rate. Employing his approach, Kramers derived the Arrhenius law [2] in its simplest form (also referred to as van't Hoff-Arrhenius, since Arrhenius built on the work of van't Hoff) for 
the reaction rate of a chemical reaction as

$$
\kappa=A \exp \left(\frac{-E}{k T}\right)
$$

where $E$ is the activation energy (a term introduced by Arrhenius) and $T$ is the temperature. $E$ is given by $V(b)-V(a)$, where $V$ denotes the potential, $a$ denotes the bottom of the well, $b$ denotes the top of the well and $A$ is the pre-exponential factor, which depends on $V^{\prime \prime}(a)$ and $\left|V^{\prime \prime}(b)\right|$, which are related to the particle's oscillation frequency at the bottom and top of the well, respectively. Note that the Arrhenius law merely states that the MFPT $\tau$ is $O\left(e^{\frac{1}{\epsilon}}\right)$, where $\epsilon=\frac{k T}{E}$. We also note that large deviation theory merely estimates the exponent in this type of law, and gives no results for the pre-exponential factor. Clearly, the higher the temperature the higher the reaction rate. Thus, milk sours faster at room temperature than in the refrigerator. More recently, there are more accurate formulas with $A$ not constant but rather dependent on temperature. The reaction process is thermally activated. The Brownian particle loses energy (e.g., via viscous drag) to the medium through which it travels (so it slows down), and the medium heats up, and vice versa. As the medium heats up, it transfers heat to the Brownian particle so that the situation is in thermal equilibrium. Since both processes stem from the collision process, it is no surprise that they are related. Thus, the coefficients of the random term in the Langevin equation $(\sqrt{2 k T})$, and of the diffusion term in the Fokker-Planck equation $(k T)$, are related, due to the fluctuation-dissipation relation. The relation must hold in order for the system to be in thermal equilibrium. Additional applications of activated processes include atomic migration in crystals, ionic conductivity in crystals, thermal fluctuations and transitions between equilibrium states in Josephson junctions, and nucleation, to name but a few.

The rate $\kappa$ then appears in the equation

$$
\frac{d C}{d t}=-\kappa C
$$

where $C$ is the concentration of a reaction component, say the limiting component.

\section{Diffusion approximation in neutron transport theory}

Above, we employed SP methods (MAE) in noisy systems. We found that the phenomenon of collisions was modelled by noise, and thus by diffusion. We now use MAE in a collision system to describe the large time approximation of the microscopic model of neutron transport by a macroscopic model - the diffusion model. It is not surprising that, as above, we obtain a diffusion equation in a collision process.

Neutron transport theory studies the evolution of a neutron population in which the neutrons can collide with the nuclei of the medium, e.g., uranium, through which they travel, and can be annihilated or can give birth to secondary neutrons upon collision. It is a subject of interest to nuclear reactor designers. The microscopic model governing neutron transport is the linear Boltzmann equation (LBE), which is a complicated model with few solutions available. Small wonder then that reactor designers would prefer to deal with simpler models, that are more amenable to analysis and whose results provide good approximations to those of LBE. One such 'approximation' in use is the diffusion model, 
though different approaches to its 'derivation' have led to different diffusion models, so that the situation is less than clear. Here, we employ SP methods to actually derive a diffusion model as an asymptotic approximation to the LBE model and bring clarity to this issue.

The nuclear age began with the discovery of the neutron by Chadwick in 1932, followed by the discovery of fission by Hahn and Meitner in 1939, and finally in 1942, with the first nuclear chain reaction in the first nuclear reactor by Fermi et al., beneath the stands of the U. Chicago's abandoned football field, as part of the Manhattan project.

The situation of interest is when there are many neutrons $\left(O\left(10^{7}\right)\right)$ per cubic $\mathrm{cm}$, and far many more $\left(O\left(10^{23}\right)\right)$ particles, e.g., nuclei, comprising the medium (the uranium). Due to the former, it is reasonable to employ a continuum theory to describe the neutron population. Due to the latter, it is important to account for neutron-nucleus collisions though safely ignoring neutron-neutron collisions, clearly because neutrons are far more likely to collide with a nucleus than with another neutron. Thus, the equation(s) governing neutron transport are linear and are referred to as the neutron transport equation or as the LBE model, in contrast to the non-LBE governing gas dynamics, which accounts for particle-particle interactions.

As a neutron travels through the medium, it suffers collisions and is likely to change direction, or it may be annihilated (absorbed), or it may result in fission, i.e., the birth of new neutrons, called secondary neutrons. In certain media, e.g., uranium, many secondary neutrons are born in the fission process. Finally, when fission occurs, a large amount of heat energy is released. This energy is then injected into a fluid which is employed for whatever desired purpose, e.g., to flow into a generator to generate electricity.

The microscopic model we employ to study the neutron population is based on the LBE integrodifferential equation

$$
\frac{1}{v} \Psi_{\tau}+\mu \Psi_{x}+\sigma(x) \Psi-\frac{\sigma(x) c(x)}{2} \int_{-1}^{1} \Psi\left(x, \mu^{\prime}, \tau\right) d \mu^{\prime}=0, \quad 0<x<d,-1 \leqslant \mu \leqslant 1, \tau>0
$$

subject to the boundary conditions

$$
\begin{aligned}
& \Psi(x=0)=f_{1}(\mu, \tau) \text { for } \mu>0, \\
& \Psi(x=d)=f_{2}(\mu, \tau) \quad \text { for } \quad \mu<0 \text {, }
\end{aligned}
$$

and an appropriate initial condition. Here, $\Psi(x, \mu, \tau)$ denotes the neutron distribution function, or the neutron density function, in a slab geometry, i.e., the probable number of neutrons at point $x$, at time $\tau$, travelling with constant speed $v$ in direction $\mu=\cos \theta$, where $\theta$ is the angle that the velocity vector makes with the horizontal. The function $\sigma(x)$ denotes the scattering cross-section, which describes the likelihood that a neutron incident on a nucleus will actually result in a scattering event (in which the direction of travel will change from $\mu^{\prime}$ to $\mu$ ), and whose average is inversely proportional to the mean free path $l$, i.e., the average distance travelled by a neutron between successive collisions. The function $c(x)$ denotes the average number of secondary neutrons born in a collision. The case $c=1$, referred to as critical, corresponds to the situation in which the 
neutron population is just sustained by the reactor. The cases $c>1(c<1)$ are referred to as supercritical (subcritical), where the neutron population grows (decays). In order to control the growth of the neutrons for safety reasons, control rods are inserted into the reactor, which absorb neutrons.

The model is based on the assumptions that (i) all neutrons travel with the same constant velocity $v$, (ii) scattering upon collision is isotropic, that is, it has no preferred directions, i.e., it is the same for all directions and (iii) the medium is source free.

The first two terms in the equation correspond to the material derivative, while the third term accounts for losses due to collisions, e.g., scattering from angle $\mu$ to angle $\mu^{\prime}$. Finally, the fourth term accounts for gains due to collisions, i.e., scattering from angle $\mu^{\prime}$ to angle $\mu$. Since we are describing the neutron density travelling in direction $\mu$, the loss term which accounts for those scattered into some other angle, we do not care about where they are scattered to, and there is only a single contribution to the loss term. In contrast, the gain term accounts for neutrons scattered from angle $\mu^{\prime}$ into angle $\mu$, and we must account for the sum of the contributions of all angles $\mu^{\prime}$, so that that term contains an integral.

Note that half a boundary condition is prescribed at each end point, corresponding to the fact that the equation contains only a first derivative in $x$ so that a total of one boundary condition should be prescribed, though there are two boundary points. Thus, we only prescribe the incoming (injected) neutrons, not those outgoing, over which we have no control. It should also be noted that the operator in the equation involves a continuous spectrum, and only a few special solutions of the problem are known. Two such approaches are the Wiener-Hopf method, and the use of so-called singular eigenfunctions (which are in fact not functions, but rather distributions). No wonder then, that engineers and scientists, interested in reactor design, say, prefer simpler theories for that purpose. The diffusion 'approximation' is such a simpler theory and has been used for design purposes.

There have been previous attempts to derive a diffusion approximation. We briefly describe two such attempts. They are referred to as the $P_{1}$ diffusion approximation and the asymptotic diffusion approximation, respectively. In the $P_{1}$ diffusion approach, the solution is first expanded in Legendre polynomials, $P_{n}(\mu)$, with the argument made that if $n$ is taken to be sufficiently large, the expansion should converge. The expansion, truncated at $n=N$, is called the $P_{N}$ approximation. In particular, if $N=1$, i.e., truncating after two terms, $P_{0}=1$ and $P_{1}=\mu$, a diffusion equation results. Note that the angular dependence of the solution is assumed, rather than derived. Of course, the question remains as to why we are allowed to truncate after only two terms. In the asymptotic diffusion approach, we are told to replace the problem posed in a finite domain by the problem posed in an infinite domain, and that rather than considering the problem originally posed with variable coefficients, we are to consider the problem with constant coefficients. Finally, we are told to consider the behaviour of the solution of this modified problem at infinity, which also leads to a diffusion equation. However, the coefficients in the two diffusion equations differ from one another, though for $c$ approaching one they approach one another. Again, questions remain why are we allowed to replace the problem originally posed in a finite domain by one in an infinite domain and why are we allowed to replace the variable coefficient problem by a constant coefficient problem? Furthermore, boundary 
conditions for both of these equations are not derived, but rather postulated in various ways.

We now present a different approach to the derivation a diffusion approximation in neutron transport theory $[18,33]$. We note that if it is to make sense as an approximation, we must be able to answer the questions: in what sense is it is an approximation, what are the conditions under which the approximation is valid, how good is the approximation and how may one provide corrections to the approximation? These questions are not answered in either approach described above.

In light of our earlier discussion of Brownian motion, it is not unreasonable to expect that the collision process in the reactor should be mathematically modelled by diffusion. However, here there are additional effects which must be accounted for, e.g., the fission and absorption processes. Finally, expectation is not equivalent to derivation.

Rather than employ a stochastic approach to a derivation of the diffusion approximation, we will only employ scaling arguments (leading to the introduction of small parameters into the problem), elementary calculus and simple SP theory, specifically, MAE.

Thus, we first non-dimensionalize the problem and introduce small parameters for the ensuing perturbation analysis. Specifically, we introduce non-dimensionalized spatial and temporal variables by

$$
y \equiv \frac{x}{d}, \quad t \equiv \frac{\bar{v} \tau}{d},
$$

where $\bar{v}$ is a reference macroscopic velocity, and introduce a non-dimensional scattering cross-section $a(y)$ by

$$
a(y)=\frac{\sigma}{\bar{\sigma}}
$$

where $\bar{\sigma}$ is a reference scattering cross-section. Finally, we introduce the small parameters

$$
\epsilon \equiv \frac{l}{d} \ll 1, \quad \delta \equiv \frac{\bar{v}}{v} \ll 1,
$$

where the former states that the mean free path $l$ is much smaller than a typical macroscopic length of the problem, e.g., the size of the reactor, and the latter states that the macroscopic velocity is much smaller than the microscopic velocity. Finally, $\Psi$ is transformed into $\psi$ and we note that the definitions above imply that $t=\epsilon \delta \tau$, which is a long time scale. We assume that $\epsilon$ and $\delta$ are of the same order of magnitude and set $\epsilon=\delta$, to get

$$
\epsilon^{2} \psi_{t}+\epsilon \mu \psi_{y}+a(y) \psi-a(y) c(y, \epsilon) \int_{-1}^{1} \psi d \mu^{\prime}=0 .
$$

This equation is clearly of SP type. We expand both $\psi$ and $c$ in asymptotic series in powers of $\epsilon$, as in the Lindstedt-Poincare method for non-linear oscillations, or bifurcation theory for non-linear BVPs. Thus,

$$
\psi \sim \sum_{n} \psi^{n}(y, t, \mu) \epsilon^{n}, \quad c \sim \sum_{n} c_{n}(y) \epsilon^{n}
$$

We insert the expansions into the model and equate each order of $\epsilon$ separately to zero, to obtain a recursive set of equations for the coefficients $\psi_{n}$ and $c_{n}$. Thus, we derive 
an outer expansion, valid only in the interior of the spatial domain, away from the boundaries. Near the boundaries, boundary layer expansions will need to be constructed. Then, by matching the outer and boundary layer expansions, we can derive boundary conditions for the derived outer equations, which will complete the derivation of the diffusion approximation. Further, the outer expansion is not expected to be valid for early times. Thus, rather than solve an initial layer problem, we note that during start-up of the reactor, additional considerations, not accounted for in the LBE model, must be accounted for. Specifically, when the power is low, $c(x)$ changes in time and that statistical fluctuations must be accounted for. Finally, they are not needed to derive the boundary conditions.

We thus obtain a sequence of equations for the recursive determination of the coefficients $\psi^{j}$ and $c_{j}$, given by

$$
L \psi^{j} \equiv a\left[\psi^{j}-\frac{c_{0}}{2} \int_{-1}^{1} \psi^{j} d \mu^{\prime}\right]=r_{j}, \quad j=0,1,2, \ldots
$$

with

$$
\begin{aligned}
& r_{0}=0 \\
& r_{1}=-\mu \psi_{y}^{0}+a \frac{c_{1}}{2} \int_{-1}^{1} \psi^{0} d \mu^{\prime} \\
& r_{2}=-\mu \psi_{y}^{1}+a \frac{c_{2}}{2} \int_{-1}^{1} \psi^{0} d \mu^{\prime}+a \frac{c_{1}}{2} \int_{-1}^{1} \psi^{1} d \mu^{\prime}-\psi_{t}^{0}
\end{aligned}
$$

We will now show that the leading term in the expansion, $\psi^{0}$, will satisfy a diffusion equation. From the equation for $\psi^{0}$, we learn that $\psi^{0}$ is independent of $\mu$, i.e., $\psi^{0}=$ $\psi^{0}(y, t)$ and $c_{0}=1$. From the equation for $\psi^{1}$, we learn that $\psi^{1}$ is linear in $\mu$, i.e., $\psi^{1}=\psi^{11}(y, t) \mu+\psi^{10}(y, t)$, that $c_{1}=0, \psi^{11}=-\frac{1}{a} \psi_{y}^{0}$ and $\psi^{10}$ can be shown to satisfy an inhomogeneous version of the diffusion equation for $\psi^{0}$. From the equation for $\psi^{2}$, we learn that $\psi^{2}$ is quadratic in $\mu$, i.e., $\psi^{2}=\psi^{22}(y, t) \mu^{2}+\psi^{21}(y, t) \mu+\psi^{20}(y, t)$, that $\psi^{22}=-\frac{1}{a} \psi_{y}^{11}$, and that $\psi^{22}+3 c_{2} \psi^{0}-\frac{3}{a} \psi_{t}^{0}=0$.

Collecting these results, we have the diffusion equation

$$
\psi_{t}^{0}=\left(\frac{1}{3 a} \psi_{y}^{0}\right)_{y}+a c_{2} \psi^{0}
$$

Note that the angular dependence of the solution (dependence on $\mu$ ) was here derived, not assumed as in the $P_{1}$ diffusion approximation.

Clearly, for $c_{2}<0$ (subcritical), the solution decays to the steady state 0 , since both terms on the r.h.s. are negative (the first term since diffusion is a negative operator), so that reaction cannot be sustained. In contrast, for $c_{2}>0$ (supercritical), the solution can approach a non-zero steady state and the reaction can be self-sustaining, as expected.

To complete the derivation of the diffusion approximation, as mentioned above, it is necessary to solve the boundary layer problem near each of the two boundary points. That is, we first stretch the spatial variable near each end point, freeze the coefficients near the relevant boundary point and solve the resulting problem on the stretched (infinite) 
domain. Then, we match the boundary layer expansion to the outer expansion, thus deriving boundary conditions for the diffusion equation. We do not carry out this exercise here, as it involves too much technical detail and is not considered in this presentation. Rather we refer the reader to the paper by Habetler and Matkowsky [18], where the boundary layer analysis and matching is carried out. By the way, our approach provides motivation for the asymptotic diffusion approach, since the boundary layer problem involves the constant coefficient problem in an infinite domain. Note too that we also derived the results that the conditions for the diffusion approximation to be valid are that $\epsilon$ must be small, i.e., the mean free path must be much smaller than all other lengths in the problem, the system must be near critical $(c \sim 1)$, and that it is valid on a long time scale.

Finally, we note that we have successfully answered the questions posed above: (i) in what sense is the approximation approximate? it is an asymptotic approximation; (ii) when is it valid? when the parameter $\epsilon$ is small, i.e., when the mean free path is much smaller than any typical macroscopic length, e.g., the size of the domain; (iii) how good is the approximation? the error is $O(\epsilon)$; (iv) how does one improve on the approximation? by including higher order terms.

\section{Conclusion}

We employed SP methods, in particular, the method of MAE and generalizations thereof, as well as the JWKB method, variational calculus and asymptotics beyond all orders, for the exit problem. The problem arises due to the effect of small random perturbations of deterministic dynamical systems, which lead to dramatic changes in the behaviour of the system. We introduced a physical argument and four different mathematical arguments to solve the exit problem, thereby resolving a long-standing problem in SP theory, namely the indeterminacy of the asymptotic expansion of the solution, sometimes referred to as the phenomenon of spurious solutions, by the standard method of MAE. We do so by either modifying or augmenting the standard method, overcoming the problem. Though results have been obtained in higher dimensions, we restricted attention to one dimension, thereby dealing with ordinary DEs, for ease and clarity of presentation so that all the ideas are clear to all.

Dedication. This lecture is dedicated to my teacher, role model, colleague and friend, Joe Keller, of blessed memory.

\section{Acknowledgements}

It is a pleasure to acknowledge my colleagues Mark Pinsky (ob'm), who introduced me to the exit problem, Elton Hsu and Mike Miksis for useful discussions. Finally, throughout my career, I have been fortunate to collaborate with extremely talented and creative people, who have been, and are, good friends. To each I am most grateful.

\section{Tam V'nishlam Shevach Lakel Boray Olam}




\section{References}

[1] Ackerberg, A. \& O'Malley, R. (1970) Boundary layer problems exhibiting resonance. Stud. Appl. Math. 49, 277-295.

[2] Arrhenius, S. (1889) Uber die Reactionsgeschwindigkeit bei der Inversion von Rohrzucker Durch Sauern. Z. Phys. Chem. 4, 226-248.

[3] Ben-Jacob, E., Bergman, D., Matkowsky, B. \& Schuss, Z. (1988) Transitions from the equilibrium state of a hysteretic Josephson junction induced by self-generated shot noise. Phys. Lett. 99A, 343-347.

[4] Ben-Jacob, E., Bergman, D., Imry, J., Matkowsky, B. \& Schuss, Z. (1983) Shot noise effect on the nonzero voltage state of the hysteretic Josephson junction. Appl. Phys. Lett. 42, $1045-1047$.

[5] Ben-Jacob, E., Bergman, Matkowsky, B. \& Schuss, Z. (1986) Master equation approach to shot noise in Josephson junctions. Phys. Rev. B 34, 1572-1581.

[6] Ben-Jacob, E., Bergman, D., Matkowsky, B. \& Schuss, Z. (1982) The lifetime of Oscillatory States. Phys. Rev. A26, 2805-2816.

[7] Brillouin, L. (1926) La Mecanique Ondulatoire de ShrodingerUne Methode Generale de Resolution par Approximations Successives. Compt. Rend. Acad. Sci. 183, 24-26.

[8] Brown, R. (1828) A brief account of microscopic observations made in the months of June, July and August contained in the pollen of plants; and on the general existence of active molecules in organic and inorganic bodies. Phil. Mag. 4, 161-173.

[9] Chapman, S. J. \& Matkowsky, B. (2013) unpublished.

[10] Dygas, M., Matkowsky, B. \& Schuss, Z. (1986) A singular perturbation approach to nonMarkovian escape rate problems. SIAM J. Appl. Math. 46, 265-298.

[11] Dygas, M., Matkowsky, B. \& Schuss, Z. (1985) On the non-Markovian theory of activated rate processes in the small friction limit. J. Chem. Phys. 83, 597-600.

[12] Dygas, M., Matkowsky, B. \& Schuss, Z. (1986) A singular perturbation approach to nonMarkovian escape rate problems with state dependent friction. J. Chem. Phys. 84, 3731-3738.

[13] Dynkin, E. (1965) Markov Processes, Springer, Berlin.

[14] Einstein, A. (1905) Uber die von der molekularkinetischen theorie der warme geforderte bewegung von in ruhenden flussigkeiten suspendierten teilichen. Ann. Phys. 17, 549-560; see also Einstern, A. (1956), Investigations on the Theory of the Brownian Movement, Ed. Furth, R., Dover Publications.

[15] FoKkeR A. (1914) Die Mittlere Energie Rotlierender Elektrische Dipole in Stralungsfeld. Ann. Phys. 43, 810-820.

[16] Friedrichs K. \& Wasow, W. (1946) Singular perturbations of nonlinear oscillations. Duke Math. J. 13, 367-381.

[17] Grasman J. \& Matkowsky, B. (1977) A variational approach to singular perturbation problems with turning points for ordinary and partial differential equations. SIAM J. Appl. Math. 32, 588-597.

[18] Habetler G. \& Matkowsky, B. (1975) Uniform asymptotic expansions in transport theory with small mean free paths. J. Mathl. Phys. 16, 846-854.

[19] Iто K. (1950) On a formula concerning stochastic differentials. Nagoya Math. J. 3, 55-65.

[20] JefrReYs, H. (1924) On certain approximate solutions of linear differential equations of the second order. Proc. London Math. Soc. 23, 428-436.

[21] Kath, W., Knessl, C. \& Matкowsky. B. (1987) Variational approach to nonlinear singularly perturbed boundary value problems. Stud. Appl. Math. 77, 61-88.

[22] Klosek-Dygas, M., Matkowsky, B. \& Schuss, Z. (1988) Colored noise in dynamical systems. SIAM J. Appl. Math. 48, 425-441.

[23] Klosek-Dygas, M, Matkowsky, B. \& Schuss, Z. (1989) Colored noise in activated rate processes. J. Stat. Phys. 54, 1309-1320.

[24] Klosek-Dygas, M., Matkowsky, B. \& Schuss, Z. (1989) First order dynamics driven by rapid Markovian jumps. SIAM J. Appl. Math. 49, 1811-1833. 
[25] Knessl, C., Matkowsky, B., Schuss, Z. \& Tier, C. (1986) A singular perturbation approach to first passage times for Markov jump processes. J. Stat. Phys. 42, 169-184.

[26] Kolmogorov A. (1931) Uber der Analytischen Methoden in Wahrscheinlichkeits Rechnung. Math. Ann. 104, 415-458.

[27] Kramers, H. (1940) Brownian motion in a field of force and the diffusion model of chemical reactions. Physica 7, 284-304.

[28] Kramers, H. (1926) Wellenmechanik und Halbzaklige Quantiszeiung. Z. Phys. 39, 828-840.

[29] Langevin, P. (1908) Sur la Theorie du Mouvement Brownien. i Comptes Rendus Acad. Sci. 146, $530-533$.

[30] LeE, J. \& WARD, M. (1994) On the asymptotic and numerical analysis of exponentially Illconditioned singularly perturbed boundary value problems. Stud. Appl. Math. 94, 271-326.

[31] Levinson, N. (1950) The first boundary value problem for $\epsilon \Delta u+A u_{x}+B u_{y}+C u=D$ for small $\epsilon$. Ann. Math. 51, 428-445.

[32] MacGillivray, A. (1997) A method for incorporating transcendentally small terms into the method of matched asymptotic expansions. Stud. Appl. Math. 99, 285-310.

[33] Matkowsky, B. (1970) unpublished.

[34] Matkowsky, B. (1975) On boundary layer problems exhibiting resonance. Siam Rev. 17, 82-100.

[35] Matkowsky, B. \& Schuss, Z. (1977) The exit problem for randomly perturbed dynamical systems. SIAM J. Appl. Math. 33, 365-382.

[36] Matkowsky, B. \& Schuss, Z. (1981) Eigenvalues of the Fokker-Planck operator and the approach to equilibrium for diffusions in potential fields. SIAM J. Appl. Math. 40, 242-254.

[37] Matkowsky, B. \& Schuss, Z. (1982) Diffusion across characteristic boundaries. SIAM J. Appl. Math. 42, 822-834.

[38] Matkowsky, B., Schuss, Z. \& Ben-Jacob, E. (1982) A singular perturbation approach to Kramers diffusion problem. SIAM J. Appl. Math. 42, 835-849.

[39] Matkowsky, B., Schuss, Z. \& Tier, C. (1983) Diffusion across characteristic boundaries with critical points. SIAM J. Appl. Math. 43, 673-695.

[40] Matkowsky, B., Schuss, Z., Knessl, C., Tier, C. \& Mangel, M. (1984) Asymptotic solution of the Kramers Moyal equation and first passage times for Markov jump processes. Phys. Rev. A29, 3359-3369.

[41] Perrin J. (1908) Mouvement brownien et Realite Moleculaire. Ann. Chim. Phys. 18, 5-114.

[42] Planck, M. (1917) Uber Einen Satz der Statischen Dynamik und Seinen Erweiterung Quantentheorie. Sitz. ber. Pruss. Akad. Wiess. 24, 324-341.

[43] Poincare, H. (1886) Sur les Integrales Irregulieres: Des Equations Lineares. Acta Math. 8, 295-344.

[44] Prandtl L. (1905) Uber Flussigkrecitsbewgung bei sehr kleiner Reibung. In Verhandlungen des 3rd International Mathematiker Kongress, Heidelberg, pp. 484-491.

[45] Schuss, Z. \& Matkowsky, B. (1979) The exit problem: A new approach to diffusion across potential barriers. SIAM J. Appl. Math. 35, 604-623.

[46] Smoluchowski, M. (1906) Zur Kinetischen Theorie und der Brownschen Molekularbewegung Suspensionen. Ann. Phys. 21, 756-780.

[47] von Kampen N. (1981) Stochastic Processes in Chemical Physics, North Holland, Amsterdam.

[48] Wasow, W. (1942)Boundary Layer Problems in the Theory of ODEs, Ph.D Thesis, NYU.

[49] Wentzel, G. (1926) Eine Verallgeneinerung der Quanten Bedingungen fur die Zwecke der Wellen Mechanek. Z. Pys. 38, 518-529.

[50] Wiener, N. (1923) Differential space. Stud. Appl. Math. 2, 131-174. 